\title{
Acute peripartum cardiomyopathy after cesarean section - A case report -
}

\author{
Departments of Anesthesiology and Pain Medicine, *Internal Medicine, \\ ${ }^{\dagger}$ Obstetrics and Gynecology, Hallym University College of Medicine, Chuncheon, Korea
}

\author{
Jae Jun Lee, M.D., Joo Sung Kim, M.D., Dong Sul Kim, M.D., Sung Mi Hwang, M.D., \\ Kyung Soon Hong, M.D.*, Eu Sun Ro, M.D. ${ }^{\dagger}$, and So Young Lim, M.D.
}

We experienced a case of acute postpartum cardiomyopathy after cesarean section. A twin-pregnant woman at 36 weeks gestation showed pregnancy-induced hypertension. After an emergency cesarean section, shortness of breath, paroxysmal dyspnea, hypertension, and tachycardia developed in the recovery room. She was diagnosed with acute peripartum cardiomyopathy. (Korean J Anesthesiol 2008; 55: 634 8)

Key Words: peripartum cardiomyopathy, pregnancy induced hypertension, twin pregnant.

Peripartum cardiomyopathy (PPCM) is a disorder of unknown cause in which initial left ventricular systolic dysfunction and symptoms of heart failure occur between the last month of pregnancy and the first 5 months postpartum. ${ }^{1,2)}$ The clinical presentation of patients with PPCM is similar to that of patients with dilated cardiomyopathy. It can be diagnosed only by the exclusion of other causes of acute heart failure such as infections, metabolic disorder, and previously asymptomatic cardiac disease exacerbated by pregnancy. The incidence of PPCM varies with individual studies and has been reported to range from 1 in 1,300 to 15,000 pregnancies. ${ }^{3,4)}$ Multiparity, twins, advanced maternal age, preeclapmsia, gestational hypertension, and black race are known risk factors. ${ }^{3,5)}$ Here, we present a case of acute postpartum cardiomyopathy after cesarean section.

\section{CASE REPORT}

A 28-year-old primigravida was diagnosed as twin pregnancy in a primary clinic. The patient visited our hospital at 14 weeks of gestation for further evaluation about high level of

Received : August 4, 2008

Corresponding to : Jae Jun Lee, Department of Anesthesiology and Pain Medicine, Hallym University College of Medicine, 153, Gyo-dong, Chuncheon 200-060, Korea. Tel: 82-33- 252-9970, Fax: 82-33-252-0941, E-mail: iloveu59@hallym.or.kr thyroid function test. She was diagnosed as hyperthyroidism and was given propylthiouracil (200 mg/day), she could keep the euthyroid state afterwards. Hypertension $(160 / 80 \mathrm{mmHg})$ with proteinuria $(++; 100-300 \mathrm{mg} / \mathrm{dL})$ was apparent at 35 weeks of gestation, diagnosed as severe preeclampsia. She gained body weight rapidly during pregnancy, and then pitting edema of the lower extremities was developed. She was recommended to enter the hospital and managed with magnesium, but she refused. At 36 weeks of gestation, she visited our hospital due to preterm labor pain, and her physician decided for emergency cesarean section. Preoperative electrocardiogram (ECG) was normal sinus rhythm, chest- $\mathrm{X}$ ray finding was within normal limits, and laboratory findings were in normal range except proteinuria $(++++; 1,000 \mathrm{mg} / \mathrm{dL} \uparrow)$. Initial vital sign in the operating room were: blood pressure, 160/110 $\mathrm{mmHg}$; heart rate, 120 beats/min; respiration, 25 breaths/min; and $\mathrm{O}_{2}$ saturation, 97\%. The ECG showed sinus tachycardia. Following infusion of normal saline $400 \mathrm{~mL}$, she was placed in the left lateral decubitus position, and combined spinal epidual (CSE) anesthesia was induced in the median approach via the L3-4 interspace using a CSE set $\left(\right.$ Espocan $\left.^{\circledR}\right)$. After tuohy needle was inserted into L3-4 epidural space by loss of resistance technique, spinal puncture was accomplished using a 27 -gauge pencil point spinal needle. The spinal puncture was accomplished smoothly at the first attempt. After obtaining a free and clear flow of cerebrospinal fluid, $7.5 \mathrm{mg}$ of hyper- 
Jae Jun Lee, et al : Peripartum cardiomyopathy after cesarean section

baric bupivacaine with $0.2 \mathrm{mg}$ of epinephrine were injected intrathecally, and epidual catheter was inserted $3 \mathrm{~cm}$ into the L3 -4 interspace. After she was turned in supine position, the blood pressure was $130 / 85 \mathrm{mmHg}$ and slowly reduced. Five minutes after anesthesia, the blood pressure had fallen to $95 / 50$ $\mathrm{mmHg}$, we injected $10 \mathrm{mg}$ ephedrine intravenously. The systolic blood pressure was maintained about $120-130 \mathrm{mmHg}$. Sensory blockade to the T4 level was confirmed by pinprick test. When the operation was started, the vital signs were: blood pressure, 120/70 $\mathrm{mmHg}$; heart rate, 100 beats/min; respiration, 20 breaths/min; $\mathrm{O}_{2}$ saturation $98 \%$. Cesarean section has

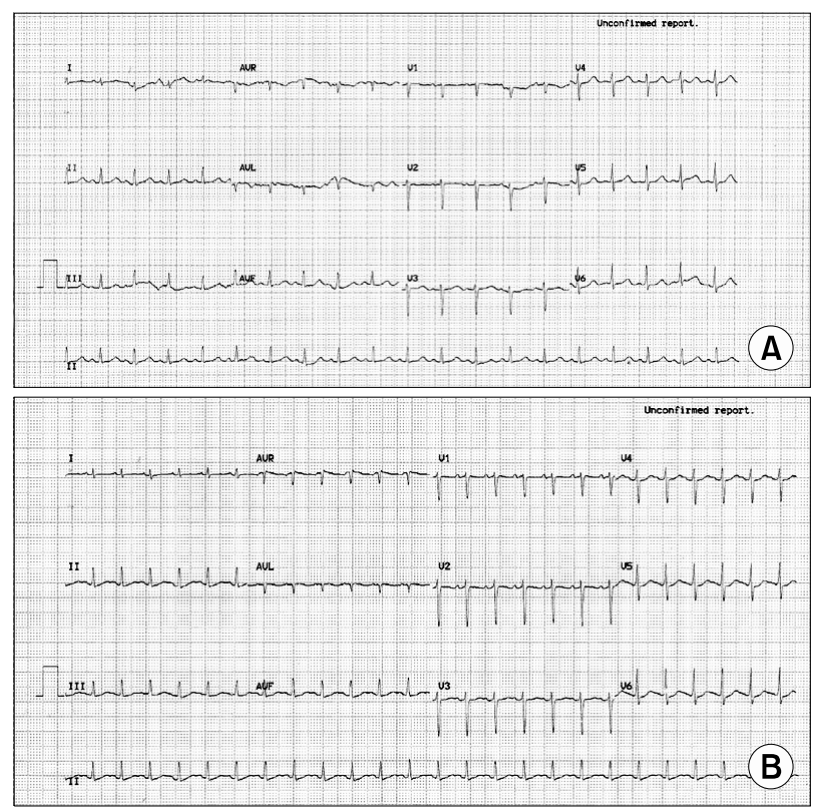

Fig. 1. Electrocardiogram (A) at the recovery room, showing sinus tachycardia, and (B) when patient complaining the chest tightness, showing sinus tachycardia with nonspecific-ST change. been done without complications, and she delivered male twin. The first neonatal body weight was $2,740 \mathrm{~g}$, one minute APGAR score was nine and five minutes APGAR score was ten. The other was $2,870 \mathrm{~g}$, nine and ten. The total operation time was 55 minutes and total administered volume was 2,050 $\mathrm{mL}$. Urine output and estimated blood loss during operation were $150 \mathrm{~mL}$ and $1,200 \mathrm{~mL}$. Her vital signs were stable throughout the operation. She was then transferred to the recovery room for further observation.

One hour after arriving the recovery room, she became short of breath, and paroxysmal dyspnea appeared, her vital signs at this time were: blood pressure, $180 / 100 \mathrm{mmHg}$; heart rate, 140 beats/min; respiration, 40 breaths $/ \mathrm{min} ; \mathrm{O}_{2}$ saturation $85 \%$. The electrocardiogram showed sinus tachycardia (Fig. 1A). Since dyspnea became more severe, intubation was done, and she was treated for pulmonary edema with oxygen, furosemide, morphine sulfate, and water restriction. Esmolol was injected intravenously for high blood pressure and tachycardia management. After a postoperative chest X-ray checked in the recovery room, she was transferred to intensive care unit (ICU), and mechanical ventilator was applied. Arterial blood gas values at $\mathrm{FiO}_{2} 1.0$ were $\mathrm{pH}, 7.32 ; \mathrm{PCO}_{2}, 32.3 \mathrm{mmHg}$; $\mathrm{PO}_{2}, 90.5 \mathrm{mmHg} ; \mathrm{HCO}^{3-} 16.8 \mathrm{mmHg}$; and $\mathrm{O}_{2}$ saturation, $96 \%$. The postoperative chest X-ray showed pulmonary edema, pleural effusion, and cardiomegaly (Fig. 2A). She was treated with furosemide and labetalol. Six hours later, her blood pressure and heart rate were normalized, and the treatment for pulmonary edema were maintained. On the second postoperative dawn, she complained of chest tightness, at that time her vital signs were: blood pressure, $154 / 108 \mathrm{mmHg}$; heart rate, 173 beats/min; respiration, 16 breaths/min; $\mathrm{O}_{2}$ saturation $88 \%$. ECG showed sinus tachycardia with nonspecific-ST change (Fig. 1B).

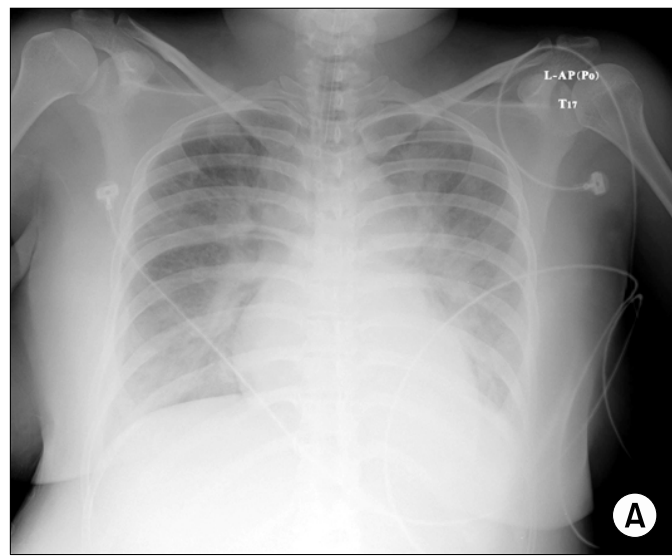

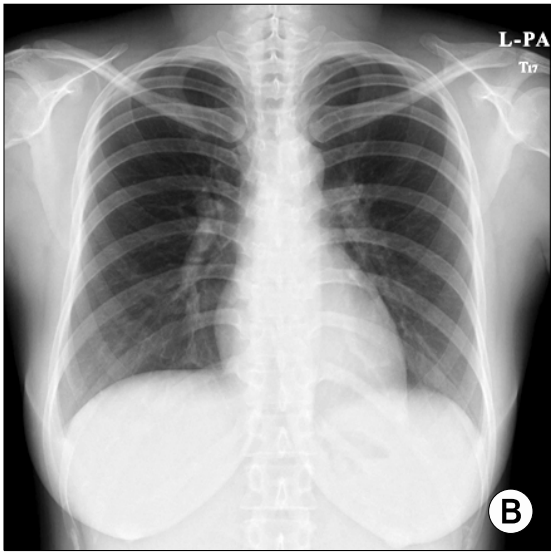

Fig. 2. Chest X-ray (A) one hour after caesarean section, showing pulmonary edema, pleural effusion with cardiomegaly, and (B) on postoperative day 12 , showing normal findings. 

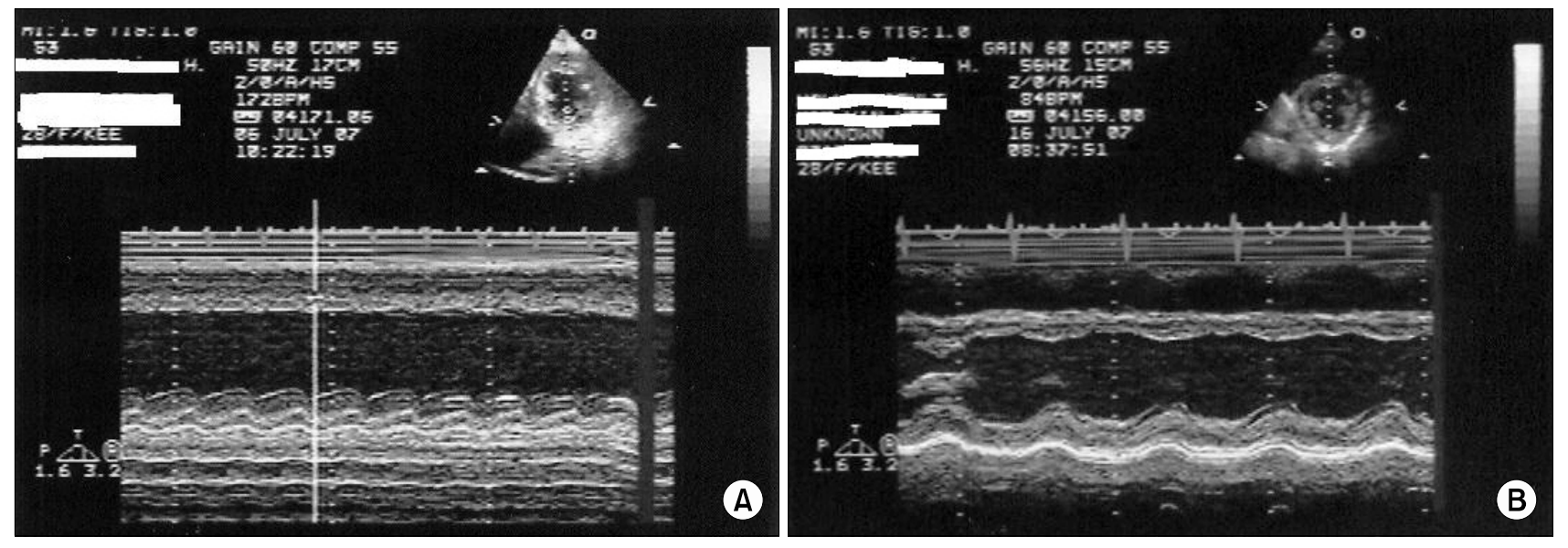

Fig. 3. M-mode echocardiogram (A) at diagnosis, showing left ventricular dilatation, severe reduction in ventricular performance, and 177 beats/min tachycardia, (B) on postoperative day 12, showing improvement in chamber dimension, normalization function, and 84 beats/min normal sinus rhythm.

Echocardiogram was performed and revealed increased left ventricular cavity size (end-diastolic diameter; $63 \mathrm{~mm}$, end-systolic diameter; $60 \mathrm{~mm}$ ), marked apical and basal part hypokinesis with mid-ventricular akinesis, severe left ventricular systolic dysfunction (ejection fraction : $<10 \%$ ), mild pericardial effusion, and both pleural effusion (Fig. 3A). Her plasma Troponin-I level was slightly high at $0.14 \mathrm{ng} / \mathrm{ml}$ (normal range $0-0.05$ $\mathrm{ng} / \mathrm{ml})$. She was diagnosed as postpartum cardiomyopathy, so that digoxin, angiotensin-converting enzyme (ACE) inhibitor, and furosemide were administrated. On postoperative day 5, pulmonary edema and pleural effusion were improved, so we decided to wean the patient from mechanical ventilation. On postoperative day 6 , echocardiogram showed left ventricular ejection fraction of $25 \%$, improved apical and basal hypokinesis, but still mid-ventricular akinesis. ECG showed T-wave inversion on lead II, III, aVF, V3-6. On postoperative day 12, chest X-ray was normalized (Fig. 2B). Echocardiogram also showed normalized left ventricular cavity size (end-diastolic diameter; $54 \mathrm{~mm}$, end-systolic diameter; $39 \mathrm{~mm}$ ), and the left ventricular ejection fraction was $45 \%$ (Fig. 3B). ECG still showed T-wave inversion on all leads except aVR. The patient was then discharged home on a regimen of ramipril, digoxin, furosemide. Three weeks after discharge, follow-up echocardiogram, chest X-ray, and ECG studies showed normal findings.

\section{DISCUSSION}

PPCM is defined as a syndrome of cardiac failure occurring between the last month of pregnancy and the first 5 months postpartum, without obvious causes and prior evidence of heart disease. The clinical presentation of patients with PPCM is similar to that of patients with dilated cardiomyopathy. It is important to recognize the association of cardiac failure and pregnancy as a separate syndrome so that, once identified, PPCM should be treated promptly and aggressively. Although a precise etiology for PPCM is unknwon, there is much current interest in infectious, autoimmune, and genetic factors that may play a role as etiologies. ${ }^{6}$ Multiparity, twins, advanced maternal age (age > 30), preeclampsia, gestational hypertension, and black race have been described as risk factors for PPCM. $^{3,5)}$ In our case, the patient had two risk factors, twins and peeclampsia. Especially, twin pregnancy has greater hemodynamic perturbations, greater hormonal change, and greater demand on nutritional reserves, all of which could be involved in the pathophysiology of PPCM.

The diagnosis of PPCM should be considered whenever a female patient presents with even mild symptoms of heart failure between the last month of pregnancy and the first 5 months postpartum. However, patients in the last months of pregnancy represent a particular diagnostic difficulty because of the common symptoms produced by pregnancy: dyspnea, fatigue and pedal edema. Early in the course of PPCM, patients may report the gradual onset of fatigue, dependent edema, orthopnea, or paroxysmal nocturnal dyspnea. ${ }^{8)}$ Symptoms and sings that should raise the suspicion of heart failure include paroxysmal nocturnal dyspnea, chest pain, nocturnal cough, new regurgitant murmurs, pulmonary crackles, elevated jugular venous pressure and hepatomegaly, ${ }^{8,9)}$ A chest X-ray study will 
Jae Jun Lee, et al : Peripartum cardiomyopathy after cesarean section

show cardiomegaly, pulmonary edema, and occasionally a small pleural effusion. ${ }^{8)}$ The electrocardiogram usually demonstrates normal sinus rhythm or sinus tachycardia. Left ventricular hypertrophy, inverted $\mathrm{T}$ waves, $\mathrm{Q}$ waves, and non-specific ST segment changes have also been reported.") Ecocardiography is now the standard non invasive technique for measuring cardiac function, quantifying left ventricular performance and providing a definitive diagnosis of left ventricular dysfunction, thus it is an important tool in properly diagnosing PPCM and predicting the prognosis. In our case, after an hour of observation at the recovery room, the patient showed paroxysmal dyspnea, hypoxemia $\left(\mathrm{SpO}_{2} 85 \%\right)$, and sinus tachycardia (150 beats/min) and cardiomegaly, pulmonary edema, and pleural effusion on the chest $\mathrm{x}$-ray. Even without chest pain, the initial patient's symptoms with ECG, and chest $x$-ray findings collectively suggest the early course of PPCM. PPCM is a rare disease and past reported cases in the anesthesiologic field in Korea were mostly cases diagnosed before cesarean section ${ }^{10,11,12)}$ and just one case which occurred during cesarean section ${ }^{13)}$ but no cases have been reported for PPCM occurring after cesarean section in the recovery room without any significant problems during the operation.

Treatment of PPCM is similar to that for other forms of heart failure. The classic treatments of congestive heart failure are bed rest, low salt intake, diuretics, digitalis, anticoagulants, suppression of lactation, and steroids. ${ }^{14)}$ The goals of treatment is reducing preload and after load, and increasing contractility. Angiotensin converting enzyme (ACE) inhibitors are usually used to reduce afterload by vasodilation, but they should be considered after delivery because of potential toxic effects on the fetus. $\beta$ blockers are used for tachycardia and arrhythmia. Digitalis may help to maximize contractility and rate control, and diuretics are used to reduce preload and relieve symptoms. ACE inhibitors and $\beta$ blockers also have been shown to improve survival in cardiomyopathy patients but no data exist for PPCM. ${ }^{15,16)}$

In half of the patients of PPCM, the cardiac size is normalized within 6 months and the patients recover without any complications. ${ }^{3,17)}$ But if congestive cardiomyopathy persist after 6 months, it is likely irreversible and portends worse survival. ${ }^{3)}$ Left ventricular enlargement and systolic dysfunction at diagnosis are prognostic indicators, and prognosis is also related to the recovery of ventricular function. In one study comparing the survivors of PPCM with non survivors, it was found that survivors had a smaller left ventricular cavity size and higher ejection fraction (58 $\mathrm{mm}$ and 23\%) at the time of diagnosis compared to non survivors $(69 \mathrm{~mm}$ and $11 \%) .{ }^{18)}$ The others reported that women who survived PPCM have higher ejection fraction and smaller left ventricular end-diastolic diameter. ${ }^{17,19)}$ In our case, the patient had large left ventricular end-diastolic diameter $(63 \mathrm{~mm})$ and severely low ejection fraction $(<10 \%)$ at the time of diagnosis. According to the references, these findings expect poor prognosis. Complete recovery of the patients, therefore, is attributed to the prompt confirmation of PPCM by echocardiography when symptom was deteriorated at the ICU and aggressive treatment. In this case, we couldn't diagnose the PPCM at the recovery room when the patient showed unexplained paroxysmal dyspnea, sinus tachycardia, and pulmonary edema. If the authors had underwent echocardiography and diagnosed PPCM at the first symptoms, more rapid recovery might have been possible.

In conclusion, if any postpartum patient has mild cough, dyspnea, sinus tachycardia on ECG, or pulmonary edema on chest x-ray, PPCM should be ruled out promptly. Delayed diagnosis can be associated with increased morbidity and mortality.

\section{REFERENCES}

1. Demakis JG, Rahimtoola SH: Peripartum cardiomyopathy. Circulation 1971; 44: 964-8.

2. Pearson GD, Veille JC, Rahimtoola S, Hsia J, Oakley CM, Hosenpud JD, et al: Peripartum cardiomyopathy. National Heart, Lung and Blood Institute and office of rare diseases (National Institutes of Health) workshop recommendations and review. JAMA 2000; 283: 1183-8.

3. Demakis JG, Rahimtoola SH, Sutton GC, Meadows WR, Szanto PB, Tobin JR, et al: Natural course of peripartum cardiomyopathy. Circulation 1971; 44: 1053-61.

4. Cunningham FG, Pritchard JA, Hankins GD, Anderson PL, Lucas MJ, Armstrong KF: peripartum heart failure: Idiopathic cardiomyopathy or compounding cardiovascular events? Obstet Gynecol 1986; 67: 157-68.

5. Meadows WR: Idiopathic myocardial failure in the last trimester of pregnancy and the puerperium. Circulation 1957; 15: 903-14.

6. Brown CS, Bertolet BD. Peripartum cardiomyopathy: a comprehensive review. Am J Obstet Gynecol 1998; 178: 409-14.

7. Reid JV: postpartal cardiomyopathy. S Afr Med J 1961; 35: 165-8.

8. Homans DC: Peripartum cardiomyopathy. N Engl J Med 1985; 312: $1432-7$.

9. Adler AK, Davis MR: Peripartum cardiomyopathy: two case reports and a review. Obstet Gynecol Surv 1986; 41: 675-82.

10. Han KR, Han JI, Jung RK, Lee GY, Lee CH: Epidural Anesthesia 
for cesarean section in pregnant woman with peripartum cardiomyopathy: a case report. Korean J Anesthesiol 1997; 32: 658-62.

11. Lee HJ, Youn HJ, Chon JY, Choi YW, Moon SH: Anesthesia for cesarean section in a parturient with peripartum cardiomyopathy: a case report. Korean J Anesthesiol 2000; 39: 278-83.

12. Park HY, Goo EK, Do SH, Park KS: Combined spinal-epidural anesthesia for cesarean section in a patient with peripartum cardiomyopathy: a case report. Korean J Anesthesiol 2007; 52: 605-8.

13. Lee BJ, Kim DO: Peripartum cardiomyopathy presenting at cesarean section: a case report. Korean J Anesthesiol 2007; 52: 359-62.

14. Veille JC: Peripartum cardiomyopathies: a review. Am J Obstet Gynecol 1984; 148: 805-18

15. SOLVD Investigators: Effects of enalapril on survival in patients with reduce left ventricular ejection fractions and congestive heart failure. N Engl J Med 1991; 325: 293-302.

16. CIBIS Investigators and Committees: A randomized trial of beta-blockade in heart failure: The Cardiac Insufficiency Bisoprolol Study (CIBIS). Circulation 1994; 90: 1765-73.

17. O'Connell JB, Costanzo-Nordin MR, Subramanian R, Robinson JA, Wallis DE, Scanlon PJ, et al: Peripartum cardiomyopathy: Clinical, hemodynamic, histologic and prognostic characteristics. J Am Coll Cardiol 1986; 8: 52-6.

18. Sutton MS, Cole P, Plappert M, Saltzman D, Goldhaber S: Effects of subsequent pregnancy on left ventricular function in peripartum cardiomyopathy. Am Heart J 1991; 121: 1776-8.

19. Aroney C, Khafagi F, Boyle C, Bett N: Peripartum cardiomyopathy: echocardiographic features in five cases. Am J Obstet Gynecol 1986; 155: 103-6. 